\title{
Parameters and fractional factors in different settings
}

\author{
Wei Gao ${ }^{1,2}$ and Juan L.G. Guirao ${ }^{1 *}$ (B)
}

"Correspondence:

juan.garcia@upct.es

1 Departamento de Matemática Aplicada y Estadística, Hospital de Marina, Universidad Politécnica de Cartagena, Cartagena, Spain

Full list of author information is available at the end of the article

\begin{abstract}
In computer networks, the feasibility of data transmission can be measured in terms of fractional factor model, and specifically the framework of fractional critical deleted graph can express the setting when certain sites and channels are unavailable at a special time. We study the relationship between some parameters in graphs and the existence of fractional $(g, f)$-factor in various settings here. Our main contributions are three-fold: first, a connectivity condition for a graph to be fractional $\left(g, f, n^{\prime}, m\right)$-critical deleted is determined; second, the relationship between independence number and fractional ID- $(g, f, m)$-deleted graphs is studied; third, an isolated toughness bound for fractional $(g, f)$-factors is given in balanced bipartite graph setting. Furthermore, by showing counterexamples we explain that bounds for parameters are tight in some sense, and corresponding conditions in all fractional factor settings are discussed as well. Finally, two open problems are proposed for future research.
\end{abstract}

MSC: $05 C 70$

Keywords: Graph; Data transmission network; Fractional factor; All fractional critical deleted graph; Fractional critical deleted graph

\section{Background and concepts}

In the mathematical modeling of computer networks, a common method is to represent the sites as vertices, and the channels between the sites as edges, so that the whole network is represented by a corresponding graph (see Trentin and Iorio [30], Nielsen and Pedersen [21], Bloem-Reddy and Orbanz [2], and Nghe et al. [20]). The direction of an edge is used to indicate the direction of a transmitted data stream. In this way, if there is a restriction of data flow from site to site, then the network corresponds to a directed graph. Instead, if there is no restriction of data flow direction (for any edge $e=u v$, the data can be transmitted both from $u$ to $v$ and from $v$ to $u$ ), then the network corresponds to an undirected graph. Therefore, when we discuss the data flow problem in the network, it's converted into the fractional flow problem in graph theory. The feasibility of data transmission in the network corresponds to the existence of fractional factors in a specific setting.

In a traditional data transmission network, the data transmission path between the stations is obtained by finding a path with the smallest weight, and the corresponding strategy is the shortest path algorithm. The limitation of this transmission approach is that when the peak period of data transmission comes, some channels can't bear the massive amount of transmission at the same time and cause congestion. At this time, other data packets

(c) The Author(s) 2019. This article is distributed under the terms of the Creative Commons Attribution 4.0 International License (http://creativecommons.org/licenses/by/4.0/), which permits unrestricted use, distribution, and reproduction in any medium, provided you give appropriate credit to the original author(s) and the source, provide a link to the Creative Commons license, and indicate if changes were made. 
can't be transmitted through the channel where the information is congested because the transmission path is determined by the shortest path selection strategy. This makes it necessary to wait for a long time for the data to pass through these channels, and eventually the transmission speed of the entire network is reduced.

In order to overcome this problem, network experts have proposed the concept of software defined networking (SDN) in recent years (see Sahoo et al. [26], Ikarashi et al. [15], Salman et al. [27], Satchou et al. [28] and Kaur et al. [16]). The most attractive feature of $\mathrm{SDN}$ is that it can calculate the data flow in the whole network in real time and select the path that can reach the destination as soon as possible, and thus avoid the congestion channels. To make a metaphor, traditional network transmission is similar to a "bus", with fixed routes and docked stations; and SDN is a "taxi", which can make route adjustments according to the road conditions at that time, avoiding the congested sections and reaching the destination the fastest. In the graph theory model, we remove the vertices corresponding to the blocked sites and the edges corresponding to the blocked channels from the original network graph, and search for the corresponding fractional factors in the remaining graphs. Therefore, the data transfer feasibility problem under the SDN framework can be converted into related problems of the fractional critical graph and the fractional deleted graph.

More extensive studies on SDN can be found in Bukhari et al. [4], Maity et al. [19], Sadik et al. [25], Shah et al. [29], and Vizarreta et al. [31].

Only simple graphs (finite, undirected, and without multi-edge and loop) are considered in our paper. Assume $G$ is a graph with vertex set $V(G)$ and edge set $E(G)$. Denote by $d_{G}(v)$ and $N_{G}(v)$ (or simply by $d(v)$ and $N(v)$ ) the degree and the neighborhood of $v \in V(G)$, respectively. Set $N_{G}[v]=N_{G}(v) \cup\{v\}$. For $S \subseteq V(G)$, we denote as $G[S]$ the subgraph of $G$ induced by $S$, and set $G-S=G[V(G) \backslash S]$. Set $e_{G}(S, T)=|\{e=u v \mid u \in S, v \in T\}|$ for two vertex-disjoint subsets $S, T \subset V(G)$. We denote the minimum degree of $G$ by $\delta(G)=\min _{v \in V(G)}\{d(v)\}$. A subset $I \subseteq V(G)$ is independent if $u v \notin E(G)$ for any $u, v \in I$. The independence number of $G$ is denoted as $\alpha(G)$ which is formulated by $\alpha(G)=\max \{|I|$ : $I$ is an independent set\}. For an incomplete graph, $S \subset V(G)$ is a vertex-cut if $G-S$ has more connected components than $G$. A vertex-cut with least cardinality is named a minimum vertex-cut of $G$, and its cardinality is the connectivity of $G$ denoted by $\kappa(G)$. The terms used without clear definitions in the article can be found in the classic book [3] written by Bondy and Murty.

Let $g$ and $f$ be nonnegative integer-valued functions defined on the vertex set of $G$ satisfying $g(v) \leq f(v)$ for any $v \in V(G)$. A fractional $(g, f)$-factor is a function $h$ defined on the edge set $E(G)$ so $h(e) \in[0,1]$ and $g(v) \leq \sum_{e \sim v} h(e) \leq f(v)$, where $e \sim v$ shows that vertex $v$ is an endpoint of edge $e$. A fractional $(g, f)$-factor is a fractional $[a, b]$-factor, a fractional $f$-factor, and a fractional $k$-factor if $g(v)=a$ and $f(v)=b$ for all $v \in V(G), g(v)=f(v)$ for all $v \in V(G)$, and $g(v)=f(v)=k(k \geq 1)$ for all $v \in V(G)$, respectively.

If for every edge subset $H \subseteq E(G)$ and $|H|=m$, a fractional $(g, f)$-factor $h$ exists so that $h(e)=0$ for any $e \in H$, then $G$ becomes a fractional $(g, f, m)$-deleted graph. Equivalently, the resulting graph admits a fractional $(g, f)$-factor after removing any $m$ edges. A graph $G$ becomes a fractional $\left(g, f, n^{\prime}\right)$-critical graph if after any $n^{\prime}$ vertices are deleted from $G$, the resulting graph admits a fractional $(g, f)$-factor. The concept of fractional $\left(g, f, n^{\prime}, m\right)$ critical deleted graph can be regarded as a combination of fractional $\left(g, f, n^{\prime}\right)$-critical graph and fractional $(g, f, m)$-deleted graph, i.e., a graph $G$ is fractional $\left(g, f, n^{\prime}, m\right)$-critical deleted 
if after $n^{\prime}$ vertices are deleted from $G$, the rest of the graph is a fractional $(g, f, m)$-deleted graph. For some recent advances on the existence of a fractional factor in various settings, we can refer to Gao et al. [7-10, 12], Liu et al. [18], and Zhou et al. [34, 35, 37, 39-43].

In this paper, we mainly study the data transmission feasibility problem in software defined networking from the view point of graph theory. The rest of the article is organized as follows. First, we introduce our main conclusions and some useful lemmas. Second, the detailed proofs are presented. Next, we show the sharpness of the given bounds. Then, the sufficient conditions in the setting of all fractional factor frameworks are discussed. Finally, several open problems are posed.

\section{Main results and useful lemmas}

Our first result reveals the relationship between connectivity and fractional $\left(g, f, n^{\prime}, m\right)$ critical deleted graphs, which improves the results given in Bian and Zhou [1], and Yuan and Hao [33].

Theorem 1 Assume $G$ is a graph, and $b, a, n^{\prime}, m, \Delta$ are nonnegative integers. Let functions $g, f$ be integer-valued on $V(G)$ satisfying $1 \leq a \leq g(x) \leq f(x)-\Delta \leq b-\Delta$ for every $x \in V(G)$. If $\kappa(G) \geq \max \left\{\frac{(b-\Delta+1)^{2}}{2}+n^{\prime}+\frac{2 m}{a+\Delta}, \frac{(b-\Delta+1)^{2} \alpha(G)+4\left((a+\Delta) n^{\prime}+2 m\right)}{4(a+\Delta)}\right\}$, then $G$ is fractional $\left(g, f, n^{\prime}, m\right)$ critical deleted.

From Theorem 1 , the connectivity condition for a graph to be fractional $\left(g, f, n^{\prime}\right)$-critical and fractional $(g, f, m)$-deleted can be obtained by taking $m=0$ and $n^{\prime}=0$, respectively. Furthermore, the connectivity condition for fractional $\left(a, b, n^{\prime}, m\right)$-critical deleted graph, fractional $\left(f, n^{\prime}, m\right)$-critical deleted graph, and fractional $\left(k, n^{\prime}, m\right)$-critical deleted graph can be determined using Theorem 1 in terms of fixing functions $g, f$ in the special settings. Here, we do not list the corollaries one by one.

A fractional ID- $(g, f, m)$-deleted graph is an unusual kind of fractional $\left(g, f, n^{\prime}, m\right)$-critical deleted graph when $n^{\prime}$ deleted vertices constitute an independent set, i.e., a graph is named as fractional independent-set-deletable $(g, f, m)$-deleted graph (shortly, fractional $I D$ - $(g, f, m)$-deleted graph) if $G-I$ is fractional $(g, f, m)$-deleted for arbitrary independent set $I \subseteq V(G)$. A fractional ID-( $(a, b, m)$-deleted graph and fractional ID-( $k, m)$-deleted graph can be defined in a special setting of functions $g$ and $f$. If $m=0$, a fractional ID$(g, f, m)$-deleted graph is simply a fractional ID- $(g, f)$-factor-critical graph.

Our second result uncovers the relationship between $\alpha(G)$ and fractional ID- $(g, f, m)$ deleted graph, which is stated as follows.

Theorem 2 Assume $G$ is a graph, and $b, a, n^{\prime}, m, \Delta$ are nonnegative integers. Let functions $g, f$ be integer-valued defined on $V(G)$ so that $1 \leq a \leq g(x) \leq f(x)-\Delta \leq b-\Delta$ for every vertex $x$. If

$$
\alpha(G) \leq \frac{4((a+\Delta)(\delta(G)-b+\Delta+1)-2 m)}{(b-\Delta+1)^{2}+4(a+\Delta)},
$$

then $G$ is fractional ID- $(g, f, m)$-deleted.

The $\alpha(G)$ condition for a graph to be fractional ID- $(g, f)$-factor-critical can be yielded by setting $m=0$ in above theorem, and the corresponding condition for fractional ID- 
$(a, b, m)$-deleted graph can be obtained by setting $f(x)=b$ and $g(x)=a$ for every $x \in V(G)$. The detailed statement of these corollaries are not provided.

Yang et al. [32] introduced isolated toughness with the function to evaluate the vulnerability of the network and denoted it as $I(G)$. For a complete graph $G$, its isolated toughness equals to $\infty$. Otherwise,

$$
I(G)=\min \left\{\frac{|S|}{i(G-S)} \mid S \subset V(G), i(G-S) \geq 2\right\},
$$

where the number of isolated vertices of $G-S$ is denoted by $i(G-S)$.

There are several articles contributing to the description of the relationship between the existence of fractional factors and isolated toughness. Li et al. [17] presented the tight isolated toughness condition for a graph to be fractional $k$-deleted. Zhou and Pan [38] proposed an isolated toughness condition for a graph to be fractional $(a, b, k)$-critical. Zhou et al. [36] put forward an isolated toughness condition for fractional $(g, f)$-factors. Gao and Wang [13] looked into the relationship between isolated toughness and a graph to be fractional $(g, f, n)$-critical. Very recently, Gao et al. [11] derived an isolated toughness condition for graphs to be fractional $(k, m)$-deleted graphs, but the sharp isolated toughness bound for a graph to be fractional $(k, m)$-deleted is still open.

A graph $G$ is called a bipartite graph if its edge set is expressed as $E(G)=\{x y \mid x \in X, y \in$ $Y$ \}, i.e., its vertex set can be divided into subsets $X$ and $Y$, and $E(G[X])=E(G[Y])=\emptyset$. When it comes to bipartite setting (as well as directed graph), the condition of factor and fractional factor can be transformed into the different versions which stated two parts, respectively (see Folkman and Fulkerson [5], and Ore [22-24] for more details).

In the bipartite graph setting, the isolated toughness can be redefined as

$$
I_{B}(G)=\min \left\{\frac{|S|}{i(G-S)}: S \subseteq X \text { or } Y, i(G-S) \geq 2\right\}
$$

where $G=(X, Y ; E(G))$ is an incomplete bipartite graph. For a complete bipartite graph $G$, $I_{B}(G)=\infty$.

Here, we present a conclusion on the relationship between isolated toughness and the existence of a fractional $(g, f)$-factor in a balanced $(|X|=|Y|)$ bipartite graph.

Theorem 3 Assume $G=(X, Y ; E(G))$ is a bipartite graph. Let $b, a, \Delta$ be three nonnegative integers with $1 \leq a \leq b-\Delta$, and functions $f$, $g$ be integer-valued on $V(G)$ satisfying $a \leq$ $g(x) \leq f(x)-\Delta \leq b-\Delta$ for every $x \in V(G)$. Let $|X|=|Y|=n>\frac{(b+a+2)(b+a-2)}{4(a+\Delta)}, f(X)=f(Y)$ and $g(X)=g(Y)$. Then $G$ admits a fractional $(g, f)$-factor if $I_{B}(G)>\frac{n-1}{2 \sqrt{(a+\Delta) n+1}-b-a}$.

The proof of our first two results depends on the lemmas below, which manifest the necessary and sufficient condition for fractional $\left(f, g, n^{\prime}, m\right)$-critical deleted graphs.

Lemma 1 (Gao [6]) Assume $G$ is a graph, functions $g, f$ are integer-valued on $V(G)$ such that $g(x) \leq f(x)$ for every vertex $x$ in $G$. Set $n^{\prime}, m$ as nonnegative integers. Then $G$ is frac- 
tional $\left(g, f, n^{\prime}, m\right)$-critical deleted graph iff

$$
\begin{aligned}
& f(S)+d_{G-S}(T)-g(T) \\
& \geq \max _{U \subseteq S, H \subseteq E(G-U),|U|=n^{\prime},|H|=m}\left\{f(U)-e_{H}(T, S)+\sum_{x \in T} d_{H}(x)\right\}
\end{aligned}
$$

for any two disjoint subsets $T, S$ of $V(G)$ with $|S| \geq n^{\prime}$.

Taking $n^{\prime}=0$, we get the sufficient and necessary condition for fractional $(g, f, m)$ deleted graphs from Lemma 1.

Lemma 2 Assume $G$ is a graph, functions $g, f$ are integer-valued on $V(G)$ with $g(x) \leq f(x)$ for every vertex $x$ in $G$. Let $m$ be a nonnegative integer and $H$ a subgraph of $G$ with $m$ edges. Then $G$ is fractional $(g, f, m)$-deleted iff

$$
f(S)+d_{G-S}(T)-g(T) \geq \sum_{x \in T} d_{H}(x)-e_{H}(T, S)
$$

for all disjoint subsets $T, S \subseteq V(G)$.

Since the necessary and sufficient condition for $(g, f)$-factors can be used in fractional $(g, f)$-factors, in light of Folkman and Fulkerson [5], we get the sufficient and necessary condition for the existence of fractional $(g, f)$-factor in the setting of bipartite graph which plays a crucial role in the proof.

Lemma 3 Assume $G=(X, Y, E(G))$ is a bipartite graph, functions $g$ and $f$ are positive integer-valued on $V(G)$ such that $g(x) \leq f(x)$ for any vertex $x$ in $G$. Then $G$ has a fractional $(g, f)$-factor iff for any $T \subseteq Y$ and $S \subseteq X$, we have

$$
f(S)+d_{G-S}(T)-g(T) \geq 0
$$

and

$$
f(T)-g(S)+d_{G-S}(S) \geq 0
$$

\section{Proof of theorems}

This section presents proofs of Theorems $1-3$ by constructing counterexamples and deducing contradiction from the necessary and sufficient conditions presented in Lemmas 1-3.

\subsection{Proof of Theorem 1}

In this subsection, we argue by contradiction to prove Theorem 1.

Assume $G$ is a graph meeting the assumption of Theorem 1 without being a fractional $\left(g, f, n^{\prime}, m\right)$-critical deleted graph. In light of Lemma 1 and $\sum_{x \in T} d_{H}(x)-e_{H}(T, S) \leq 2 m$, there exist disjoint subsets $S$ and $T$ of $V(G)$ so that

$$
(\Delta+a)\left(|S|-n^{\prime}\right)+d_{G-S}(T)-(b-\Delta)|T| \leq f(S-U)+d_{G-S}(T)-g(T) \leq 2 m-1,
$$


where $U \subseteq S$ with $|S| \geq|U|=n^{\prime} ; S$ and $T$ are selected with minimum $|T|$. Therefore, for every $x \in T$, we obtain $d_{G-S}(x) \leq g(x)-1 \leq b-1-\Delta$. If some $x \in T$ exists to satisfy $d_{G-S}(x) \geq g(x) \geq b-\Delta, S$ and $T \backslash\{x\}$ also satisfies (2), we get a contradiction to the choice of $T$ and $S$.

If $|T|=0$, then $\sum_{x \in T} d_{H}(x)-e_{H}(T, S)=0$, and we immediately get a contradiction using Lemma 1 again. Thus, $T \neq \emptyset$.

Set $T_{1}=G[T]$, which is the subgraph induced by $T$. Select $v_{1} \in T_{1}$ satisfying $d_{T_{1}}\left(v_{1}\right)=$ $\delta\left(T_{1}\right)$ and set $L_{1}=N_{T_{1}}\left[v_{1}\right]$. For $i \geq 2$, select $v_{i} \in T_{i}=T_{1}-\bigcup_{1 \leq j<i} L_{j}$ with $d_{T_{i}}\left(v_{i}\right)=\delta\left(T_{i}\right)$ and $L_{i}=N_{T_{i}}\left[v_{i}\right]$. Set $\left|L_{i}\right|=d_{i}$. Repeat the procedure until $T_{i}=\emptyset$ for some $i$; assume $i=r+1$. Thus, $\left\{v_{1}, v_{2}, \ldots, v_{r}\right\} \subseteq V(G)$ is an independent set. Furthermore, we get $r \geq 1$ and $T=$ $\sum_{1 \leq i \leq r} d_{i}$. Set $U=V(G) \backslash(S \cup T)$ and $\kappa(G-S)=t$.

Next, we provide some useful claims.

Claim $1 \frac{(a+b+1)^{2}-4}{4(a+\Delta)} \leq \frac{(b-\Delta+1)^{2}}{2}$.

Proof of Claim 1 It is enough to prove $2(\Delta+a)(b+1-\Delta)^{2}+4-(b+a+1)^{2} \geq 0$. Let $a+\Delta=x_{1} \geq 1$ and $b-\Delta+1=x_{2} \geq 2$. Then, $2(a+\Delta)(b-\Delta+1)^{2}-(a+b+1)^{2}+4=$ $2 x_{1} x_{2}^{2}-\left(x_{1}+x_{2}\right)^{2}+4=2 x_{1} x_{2}^{2}-x_{1}^{2}-x_{2}^{2}-2 x_{1} x_{2}+4$. Set

$$
\Psi\left(x_{2}\right)=2 x_{1} x_{2}^{2}-x_{1}^{2}-x_{2}^{2}-2 x_{1} x_{2}+4=\left(2 x_{1}-1\right) x_{2}^{2}-2 x_{1} x_{2}-x_{1}^{2}+4
$$

Note that $2 x_{1}-1 \geq 1$, and so when $x_{2}=\frac{x_{1}}{2 x_{1}-1}, \Psi\left(x_{2}\right)$ reaches the minimum value. Furthermore, $\Psi\left(x_{2}\right)$ is monotonically increasing when $x_{2}>\frac{x_{1}}{2 x_{1}-1}$. However, $x_{2} \geq 2$ and $\frac{x_{1}}{2 x_{1}-1} \leq 1$. Hence, since $x_{1} \geq 1$, we have

$$
\Psi\left(x_{2}\right) \geq \Psi(2)=-x_{1}^{2}+4 x_{1}>0 .
$$

Thus, the proof of Claim 1 is finished.

Claim $2 U \neq \emptyset$ or $r \geq 2$.

Proof of Claim 2 Suppose that $r=1$ and $U=\emptyset$. Using (2), we have

$$
\begin{aligned}
(a+\Delta)\left(|S|-n^{\prime}\right)+d_{1}\left(d_{1}-1\right)-(b-\Delta) d_{1} \\
\quad=(a+\Delta)\left(|S|-n^{\prime}\right)+d_{G-S}(T)-(b-\Delta)|T| \leq 2 m-1,
\end{aligned}
$$

which implies

$$
|S| \leq \frac{-d_{1}^{2}+d_{1}+(b-\Delta) d_{1}+2 m-1}{a+\Delta}+n^{\prime} .
$$

By means of Claim 1, we infer

$$
\begin{aligned}
|V(G)| & =|S|+d_{1} \leq \frac{-d_{1}^{2}+d_{1}+(b-\Delta) d_{1}+2 m-1}{a+\Delta}+n^{\prime}+d_{1} \\
& =\frac{-d_{1}^{2}+d_{1}+(b-\Delta) d_{1}+(\Delta+a) d_{1}}{\Delta+a}+n^{\prime}+\frac{2 m-1}{\Delta+a}
\end{aligned}
$$




$$
\begin{aligned}
& =\frac{-d_{1}^{2}+d_{1}(b+a+1)}{\Delta+a}+n^{\prime}+\frac{2 m-1}{\Delta+a} \\
& \leq \frac{(b+a+1)^{2}}{4(\Delta+a)}+n^{\prime}+\frac{2 m-1}{\Delta+a} \\
& \leq \frac{(b+1-\Delta)^{2}}{2}+n^{\prime}+\frac{2 m}{\Delta+a},
\end{aligned}
$$

which contradicts the fact that $|V(G)|>\kappa \geq \frac{(b+1-\Delta)^{2}}{2}+\frac{2 m}{a+\Delta}+n^{\prime}$.

Claim $3 \sum_{v \in T} d_{G-S}(v) \geq \sum_{1 \leq i \leq r}\left(d_{i}^{2}-d_{i}\right)+\frac{r t}{2}$.

The proof of Claim 3 can be found in the work of Yuan and Hao [33].

In light of Claim 3, $\frac{(b-\Delta+1)^{2}}{4}+d_{i}^{2} \geq(b-\Delta+1) d_{i}$, and, due to the fact that $|T|=\sum_{1 \leq i \leq r} d_{i}$, we deduce

$$
\begin{aligned}
2 m-1 & \geq\left(|S|-n^{\prime}\right)(\Delta+a)+d_{G-S}(T)-(b-\Delta)|T| \\
& \geq\left(|S|-n^{\prime}\right)(\Delta+a)+\sum_{1 \leq i \leq r}\left(d_{i}^{2}-d_{i}\right)+\frac{r t}{2}-(b-\Delta)|T| \\
& =(a+\Delta)\left(|S|-n^{\prime}\right)+\sum_{1 \leq i \leq r} d_{i}\left(d_{i}-1\right)+\frac{r t}{2}-(b-\Delta) \sum_{1 \leq i \leq r} d_{i} \\
& =(a+\Delta)\left(|S|-n^{\prime}\right)+\sum_{1 \leq i \leq r}\left(d_{i}^{2}-(b-\Delta+1) d_{i}\right)+\frac{r t}{2} \\
& \geq(a+\Delta)\left(|S|-n^{\prime}\right)-\frac{(b-\Delta+1)^{2} r}{4}+\frac{r t}{2} .
\end{aligned}
$$

Claim $4-\frac{(b-\Delta+1)^{2}}{4}+\frac{t}{2}<0$

The proof of Claim 4 can be found in the work of Bian and Zhou [1].

In view of (2), Claims 3-4, $\alpha(G) \geq \alpha(G[T]) \geq r$, and the condition of Theorem 1, we get

$$
\begin{aligned}
2 m-1 \geq & (a+\Delta)\left(|S|-n^{\prime}\right)-\frac{(b-\Delta+1)^{2}}{4} r+\frac{r t}{2} \\
= & (a+\Delta)\left(|S|-n^{\prime}\right)+\left(-\frac{(b+1-\Delta)^{2}}{4}+\frac{t}{2}\right) r \\
\geq & (a+\Delta)\left(\kappa(G)-t-n^{\prime}\right)+\left(-\frac{(b+1-\Delta)^{2}}{4}+\frac{t}{2}\right) \alpha(G) \\
\geq & (\Delta+a)\left(\kappa(G)-t-n^{\prime}\right)+\left(-\frac{(b+1-\Delta)^{2}}{4}+\frac{t}{2}\right) \\
& \times \frac{4(\Delta+a) \kappa(G)-4\left((\Delta+a) n^{\prime}+2 m\right)}{(b-\Delta+1)^{2}} \\
= & (a+\Delta) t\left(\frac{2 \kappa(G)-2 n^{\prime}}{(b-\Delta+1)^{2}}-1\right)+2 m-\frac{4 m t}{(b-\Delta+1)^{2}} \\
\geq & (a+\Delta) t\left(\frac{2\left(\frac{2 m}{a+\Delta}+n^{\prime}+\frac{(b+1-\Delta)^{2}}{2}\right)-2 n^{\prime}}{(b+1-\Delta)^{2}}-1\right)+2 m-\frac{4 m t}{(b+1-\Delta)^{2}} \\
= & 2 m,
\end{aligned}
$$

a contradiction. 
Thus, we complete the proof of Theorem 1.

\subsection{Proof of Theorem 2}

Assume $I \subset V(G)$ is an independent set and $G^{\prime}=G-I$. Clearly, we have $\delta\left(G^{\prime}\right) \geq \delta(G)-|I|$, and Theorem 2 holds if and only if $G^{\prime}$ is fractional $(g, f, m)$-deleted. To the contrary, we assume that $G^{\prime}$ is not a fractional $(g, f, m)$-deleted graph. In view of Lemma 2 and $\sum_{x \in T} d_{H}(x)-e_{H}(T, S) \leq 2 m$ for any subset $H$ with $|E(H)|=m$, non-disjoint subsets $S, T \subseteq V\left(G^{\prime}\right)$ exist, satisfying

$$
|S|(\Delta+a)+d_{G^{\prime}-S}(T)-(b-\Delta)|T| \leq f(S)-g(T)+d_{G^{\prime}-S}(T) \leq 2 m-1 .
$$

Choose $T$ and $S$ with minimum $|T|$, and we have $d_{G^{\prime}-S}(x) \leq b-1-\Delta$ for each $x \in T$ and $|T| \neq 0$ (if $T=\emptyset$, then $\sum_{x \in T} d_{H}(x)-e_{H}(T, S)=0$, and a contradiction is obtained via Lemma 2 again).

Assume $d_{1}=\min \left\{d_{G^{\prime}-S}(x): x \in T\right\}$ and select $v_{1} \in T$ having $d_{G^{\prime}-S}\left(v_{1}\right)=d_{1}$. We obtain $0 \leq d_{1} \leq b-\Delta-1$. Now, we present a lower bound of $|S|$ with regard to $\delta(G)$ and $\alpha(G)$.

Claim $5|S| \geq \delta(G)-\alpha(G)-d_{1}$.

Proof of Claim 5 Obviously,

$$
\delta\left(G^{\prime}\right) \leq d_{G^{\prime}}\left(v_{1}\right) \leq d_{G^{\prime}-S}\left(v_{1}\right)+|S|=d_{1}+|S|
$$

which implies $|S| \geq \delta\left(G^{\prime}\right)-d_{1}$. Combining this fact with $\delta\left(G^{\prime}\right) \geq \delta(G)-|I|$ yields

$$
|S| \geq \delta(G)-|I|-d_{1}
$$

From (4) and $|I| \leq \alpha(G)$, we get the desired claim.

Consider the subgraph $T_{1}=G^{\prime}[T]$. The following procedure is similar to that in Sect. 3.1. Select $v_{1} \in T_{1}$ which satisfies $d_{T_{1}}\left(v_{1}\right)=\delta\left(T_{1}\right)$ and set $L_{1}=N_{T_{1}}\left[v_{1}\right]$. For $i \geq 2$, select $v_{i} \in$ $T_{i}=T_{1}-\bigcup_{1 \leq j<i} L_{j}$ with $d_{T_{i}}=\delta\left(T_{i}\right)$ and $L_{i}=N_{T_{i}}\left[v_{i}\right]$. Set $\left|L_{i}\right|=d_{i}$. Repeat the procedure until $T_{i}=\emptyset$ for some $i$; assume $i=r+1$. Thus, $\left\{v_{1}, v_{2}, \ldots, v_{r}\right\} \subseteq V(G)$ is an independent set. Furthermore, we get $r \geq 1, \alpha\left(G^{\prime}[T]\right) \geq r$ and $T=\sum_{1 \leq i \leq r} d_{i}$.

Due to $\alpha(G) \geq \alpha(G[T])=\alpha\left(G^{\prime}[T]\right)$, we derive

$$
\alpha(G) \geq r
$$

Inspired by Claim 3 in Sect. 3.1, we prove the claim below.

Claim $6 d_{G^{\prime}-S}(T) \geq \sum_{1 \leq i \leq r}\left(d_{i}^{2}-d_{i}\right)$.

Proof of Claim 6 Since the choice of $v_{i}$ demonstrates that each vertex in $L_{i}$ has degree at least $d_{i}-1$ in $T_{i}$, we infer

$$
\sum_{1 \leq i \leq r}\left(\sum_{x \in L_{i}} d_{T_{i}}(x)\right) \geq \sum_{1 \leq i \leq r}\left(d_{i}^{2}-d_{i}\right) .
$$


Thus, we have

$$
d_{G^{\prime}-S}(T) \geq \sum_{1 \leq i \leq r}\left(d_{i}^{2}-d_{i}\right)+\sum_{1 \leq i<j \leq r} e_{G^{\prime}}\left(L_{i}, L_{j}\right) \geq \sum_{1 \leq i \leq r}\left(d_{i}^{2}-d_{i}\right),
$$

and so we complete the proof of Claim 6.

In light of Claims 5-6, the results deduced above, the assumption

$$
\alpha(G) \leq \frac{4((a+\Delta)(\delta(G)-b+\Delta+1)-2 m)}{(b-\Delta+1)^{2}+4(a+\Delta)}
$$

and the fact that $d_{i}^{2}-(b-\Delta+1) d_{i} \geq-\frac{(b-\Delta+1)^{2}}{4}$, we obtain

$$
\begin{aligned}
2 m-1 \geq & (a+\Delta)|S|-(b-\Delta)|T|+d_{G^{\prime}-S}(T) \\
\geq & (a+\Delta)\left(\delta(G)-d_{1}-\alpha(G)\right)-(b-\Delta) \sum_{1 \leq i \leq r} d_{i}+\sum_{1 \leq i \leq r}\left(d_{i}^{2}-d_{i}\right) \\
= & (a+\Delta)\left(\delta(G)-d_{1}-\alpha(G)\right)+\sum_{1 \leq i \leq r}\left(d_{i}^{2}-(b-\Delta+1) d_{i}\right) \\
\geq & (a+\Delta)\left(\delta(G)-d_{1}-\alpha(G)\right)-\sum_{1 \leq i \leq r} \frac{(b-\Delta+1)^{2}}{4} \\
= & (a+\Delta)\left(\delta(G)-d_{1}-\alpha(G)\right)-r \frac{(b+1-\Delta)^{2}}{4} \\
\geq & (a+\Delta)\left(\delta(G)-d_{1}-\alpha(G)\right)-\alpha(G) \frac{(b+1-\Delta)^{2}}{4} \\
= & (a+\Delta)\left(\delta(G)-d_{1}\right)-\frac{4(a+\Delta)+(b+1-\Delta)^{2}}{4} \alpha(G) \\
\geq & (a+\Delta)(\delta(G)-b+1+\Delta) \\
& -\frac{(b+1-\Delta)^{2}+4(a+\Delta)}{4} \frac{4((a+\Delta)(\delta(G)-b+\Delta+1)-2 m)}{(b-\Delta+1)^{2}+4(a+\Delta)} \\
= & 2 m, \quad
\end{aligned}
$$

a contradiction. Thus, the desired conclusion follows.

\subsection{Proof of Theorem 3}

The main purpose in this section is to prove the result in the bipartite graph setting.

We suppose that a bipartite graph $G$ satisfies the assumption in Theorem 3, without a fractional $(g, f)$-factor. By Lemma $3, S \subseteq X$ and $T \subseteq Y$ exist satisfying

$$
f(S)+d_{G-S}(T)-g(T)<0
$$

or

$$
f(T)-g(S)+d_{G-S}(S)<0 .
$$


Since the status of $X$ and $Y$ in the bipartite graph is symmetrical, we assume (6) holds (the case when (7) holds can be discussed similarly). We have

$$
d_{G-S}(T) \leq g(T)-1-f(S) \leq(b-\Delta)|T|-(a+\Delta)|S|-1 .
$$

The discussion below is divided into two parts based on the relationship between $|S|+|T|$ and $n$.

Case 1. $|S|+|T| \leq n$.

Let $y_{0} \in T$ be the vertex adjacent to the least number (this number is denoted by $d$ ) of vertices in $X-S$. According to (8), we have $d|T| \leq(b-\Delta)|T|-1-|S|(a+\Delta)$ and then $|S| \leq \frac{(b-\Delta-d)|T|-1}{a+\Delta}$. Combining with $|S|+|T| \leq n$, we get $|S| \leq \frac{(b-\Delta-d) n-1}{a+b-d}$. Set $T^{\prime}=Y-\left\{y_{0}\right\}$. We obtain $\left|T^{\prime}\right|=n-1$ and $i\left(G-T^{\prime}\right) \geq n-d-|S| \geq n-\frac{(b-\Delta-d) n-1}{a+b-d}-d=b+a-d+\frac{(a+\Delta) n+1}{a+b-d}-$ $b-a \geq 2 \sqrt{(a+\Delta) n+1}-b-a>0$. Therefore, we infer

$$
I_{B}(G) \leq \frac{\left|T^{\prime}\right|}{i\left(G-T^{\prime}\right)} \leq \frac{n-1}{n-d-|S|} \leq \frac{n-1}{2 \sqrt{(\Delta+a) n+1}-b-a},
$$

a contradiction.

Case 2. $|T|+|S|>n$.

Set $T^{\prime}=Y-T$ and $S^{\prime}=X-S$. We have $\left|S^{\prime}\right|+\left|T^{\prime}\right|<n$. In light of $d_{G-S}(T)=d_{G-T^{\prime}}\left(S^{\prime}\right)$ and (8), we deduce

$$
\begin{aligned}
d_{G-T^{\prime}}\left(S^{\prime}\right) & \leq g(T)-1-f(S)=g\left(Y-T^{\prime}\right)-1-f\left(X-S^{\prime}\right) \\
& =g(Y)+f\left(S^{\prime}\right)-f(X)-1-g\left(T^{\prime}\right) \\
& \leq f\left(S^{\prime}\right)-1-g\left(T^{\prime}\right) \\
& \leq(b-\Delta)\left|S^{\prime}\right|-(a+\Delta)\left|T^{\prime}\right|-1 .
\end{aligned}
$$

If $\left|T^{\prime}\right|=0$, then $d_{G-T^{\prime}}\left(S^{\prime}\right) \geq b\left|S^{\prime}\right|$ contradicts the above inequality. Hence, $\left|T^{\prime}\right| \geq 1$. Similarly, we have $\left|T^{\prime}\right| \leq n-1$. Now we exchange $|T|$ and $|S|$ with $\left|S^{\prime}\right|$ and $\left|T^{\prime}\right|$, respectively. Using the same arguments as in Case 1 (i.e., when $|S|+|T| \leq n$ ), we can obtain $I_{B}(G) \leq \frac{n-1}{2 \sqrt{(\Delta+a) n+1-b-a}}$ which contradicts the assumption of Theorem 3.

\section{Sharpness}

In this section, we analyze the sharpness of our results.

\subsection{Tightness of $\kappa(G)$ condition in Theorem 1}

First, let's show that the condition $\kappa(G) \geq \frac{(b-\Delta+1)^{2}}{2}+\frac{2 m}{a+\Delta}+n^{\prime}$ can't be replaced by $\frac{(b-\Delta+1)^{2}}{2}+\frac{2 m}{a+\Delta}+n^{\prime}-1$. Let $b, a, n^{\prime}, m, \Delta$ be five integers with $1 \leq b-\Delta=a$, and $G=K_{\frac{(b+1-\Delta)^{2}}{2}+n^{\prime}+\frac{2 m}{a+\Delta}-1} \vee \frac{\left((b+1-\Delta)^{2}-2\right) b+2+4 m}{2 a} K_{1}$ where both $\frac{b\left((b+1-\Delta)^{2}-2\right)+2+4 m}{2 a}$ and $\frac{(b-\Delta+1)^{2}}{2}+$ $\frac{2 m}{a+\Delta}+n^{\prime}-1$ are integers. Set $S=V\left(K_{\frac{(b+1-\Delta)^{2}}{2}+n^{\prime}+\frac{2 m}{a+\Delta}-1}\right)$ and $T=V\left(\frac{b\left((b+1-\Delta)^{2}-2\right)+4 m+2}{2 a} K_{1}\right)$. Set $f(x)=b=a+\Delta$ and $g(x)=a$ for all vertices $x$ in $G$. We have $\kappa(G)=\frac{(b-\Delta+1)^{2}}{2}+n^{\prime}+\frac{2 m}{a}-1>n^{\prime}$, $|S|=\frac{(b-\Delta+1)^{2}}{2}+n^{\prime}+\frac{2 m}{a}-1,|T|=\frac{b\left((b-\Delta+1)^{2}-2\right)+4 m+2}{2 a}$, and $d_{G-S}(T)=0$. Let $U \subset S, H \subseteq$ $E(G-U)$ with $|U|=n^{\prime}$ and $|H|=m$. One can check that $e_{H}(T, S)=\sum_{x \in T} d_{H}(x)$. Therefore, 
we obtain

$$
\begin{aligned}
f & (S-U)-g(T)+d_{G-S}(T)-\left(\sum_{x \in T} d_{H}(x)-e_{H}(T, S)\right) \\
& =\left(|S|-n^{\prime}\right) b-a|T|+d_{G-S}(T) \\
& =b\left(\frac{(b-\Delta+1)^{2}}{2}+\frac{2 m}{a+\Delta}-1\right)-a \frac{b\left((b-\Delta+1)^{2}-2\right)+4 m+2}{2 a} \\
& =-1 .
\end{aligned}
$$

By means of Lemma $1, G$ is not fractional $\left(g, f, n^{\prime}, m\right)$-critical deleted.

The condition $\kappa(G) \geq \frac{(b-\Delta+1)^{2} \alpha(G)+4\left((a+\Delta) n^{\prime}+2 m\right)}{4(a+\Delta)}=\frac{\left(\frac{b-\Delta+1}{2}\right)^{2} \alpha(G)+(a+\Delta) n^{\prime}+2 m}{a+\Delta}$ in Theorem 1 is best possible, since it can't be replaced by $\frac{\left(\frac{b-\Delta+1}{2}\right)^{2} \alpha(G)+(a+\Delta) n^{\prime}+2 m-1}{a+\Delta}$. Let $G=$ $K_{\left.\frac{(-\underline{-}-\underline{1}+1}{2}\right)^{2} r+(a+\Delta) n^{\prime}+2 m-1} \vee\left(r K_{\frac{b-\Delta+1}{2}}\right)$, where $b, a, n^{\prime}, m, \Delta$ are nonnegative integers with $1 \leq b-\Delta \stackrel{a+\Delta}{=} a, r$ is a large integer, and both $\frac{\left(\frac{b-\Delta+1}{2}\right)^{2} r+(a+\Delta) n^{\prime}+2 m-1}{a+\Delta}$ and $\frac{b-\Delta+1}{2}$ are integers. It's checked that $\alpha(G)=r$ and $\kappa(G)=\frac{\left(\frac{b-\Delta+1}{2}\right)^{2} r+(a+\Delta) n^{\prime}+2 m-1}{a+\Delta}$. Let $f(x)=\Delta+a=b$ and $g(x)=a$ for all vertices $x$ in $G$. Set $T=V\left(r K_{\frac{b-\Delta+1}{2}}\right), S=V\left(K_{\left.\frac{(b+1-\Delta}{2}\right)^{2} r+(\Delta+a) n^{\prime}-1+2 m}\right)$, and let $U \subset S$ and $H \subseteq E(G-U)$ be such that $|U|=n^{\prime}$ and $|H|=m$. Using $\sum_{x \in T}^{a+\Delta} d_{H}(x)-e_{H}(T, S) \leq 2 m$ for any edge set $H$ in $G-U$, we infer

$$
\begin{aligned}
f(S & -U)-g(T)+d_{G-S}(T)-\left(\sum_{x \in T} d_{H}(x)-e_{H}(T, S)\right) \\
& \geq\left(|S|-n^{\prime}\right) b-a|T|+d_{G-S}(T)-2 m \\
& =\frac{\left(\frac{b-\Delta+1}{2}\right)^{2} r+2 m-1}{\Delta+a} b+\frac{(b+1-\Delta) r}{2}\left(\frac{b+1-\Delta}{2}-1\right)-a \frac{(b+1-\Delta) r}{2}-2 m \\
& =-1 .
\end{aligned}
$$

Then, in view of Lemma 1 , we confirm that $G$ is not fractional $\left(g, f, n^{\prime}, m\right)$-critical deleted.

\subsection{Tightness of $\alpha(G)$ condition in Theorem 2}

Now, we illustrate the sharpness of Theorem 2. Let $G=K_{t} \vee(t+1) K_{b+1}$ where $t=$ $\left\lfloor\frac{4((a+\Delta)(\delta(G)-b+\Delta+1)-2 m)}{(b+1-\Delta)^{2}+4(\Delta+a)}\right\rfloor$ is an integer (since $\delta(G)=b+t$, and we can get the detailed expression of $t$ according to specific $a, b$ and $\Delta$, if $\Delta$ is large enough and $m$ is small enough). We confirm that

$$
\begin{aligned}
& \frac{4((a+\Delta)(\delta(G)-b+1+\Delta)-2 m)}{(b+1-\Delta)^{2}+4(\Delta+a)} \\
& <\alpha(G)=t+1 \\
& =\left\lfloor\frac{4((\Delta+a)(\delta(G)-b+1+\Delta)-2 m)}{(b+1-\Delta)^{2}+4(\Delta+a)}\right\rfloor+1 \\
& \leq \frac{4((\Delta+a)(\delta(G)-b+1+\Delta)-2 m)}{(b+1-\Delta)^{2}+4(\Delta+a)}+1 .
\end{aligned}
$$

We take a vertex $v_{i}(1 \leq i \leq t+1)$ in each $K_{b+1}$ and set $I=\left\{v_{1}, v_{2}, \ldots, v_{t+1}\right\}$. Hence, $I$ is an independent set of $G$ and $G^{\prime}=G-I=K_{t} \vee(t+1) K_{b}$. Set $S=V\left(K_{t}\right), T=V\left((t+1) K_{b}\right), f(x)=$ 
$b=g(x)$ for each $x \in V(T)$ and $f(x)=a=g(x)$ if $x \in V(S)$. We obtain $|S|=t,|T|=(t+1) b$, and $d_{G^{\prime}-S}(T)=b(t+1)(b-1)$. Consider $H \subseteq E(G[T])$ with $m$ edges and $\sum_{x \in T} d_{H}(x)-$ $e_{H}(T, S)=2 m$. Thus, we infer

$$
\begin{aligned}
f(S) & -g(T)+d_{G-S}(T)-\left(\sum_{x \in T} d_{H}(x)-e_{H}(T, S)\right) \\
= & a|S|-b|T|+d_{G-S}(T)-2 m \\
= & a t-(t+1) b^{2}+b(t+1)(b-1)-2 m \\
= & a t-b t-b-2 m<0 .
\end{aligned}
$$

In terms of Lemma $2, G^{\prime}$ is not fractional $(g, f, m)$-deleted, and thus $G$ is not fractional ID- $(g, f, m)$-deleted.

\section{Discussions about all fractional factor settings}

It's said that there are all fractional $(g, f)$-factors in $G$ if $G$ has a fractional $p$-factor for each $p: V(G) \rightarrow \mathbb{N}$ with $g(v) \leq p(v) \leq f(v)$ for any $v \in V(G)$. If $g(v)=a, f(v)=b$ for each vertex $v$ and $G$ has all fractional $(g, f)$-factors, then $G$ has all fractional $[a, b]$-factors. A graph $G$ is an all fractional $(g, f, m)$-deleted graph if after any $m$ edges deleted, the resulting graph has all fractional $(g, f)$-factors.

A graph $G$ is an all fractional $\left(g, f, n^{\prime}, m\right)$-critical deleted graph if after any $n^{\prime}$ vertices of $G$ deleted, the remaining graph is an all fractional $(g, f, m)$-deleted graph. Set $g(v)=a$, $f(v)=b$ for each $v \in V(G)$, then all fractional $\left(g, f, n^{\prime}, m\right)$-critical deleted graph becomes all fractional $\left(a, b, n^{\prime}, m\right)$-critical deleted graph. Currently, Gao et al. [14] has put forward the necessary and sufficient condition for a graph to be all fractional $\left(g, f, n^{\prime}, m\right)$-critical deleted.

Lemma 4 (Gao et al. [14]) Assume $m$ and $n^{\prime}$ are nonnegative integers. Set $g, f: V(G) \rightarrow \mathbb{Z}^{+}$ as integer-valued functions with $g(x) \leq f(x)$ for every vertex $x$ in $G$, and $H$ as a subgraph of $G$ having $m$ edges. Then $G$ is all fractional $\left(g, f, n^{\prime}, m\right)$-critical deleted iff

$$
g(S)+\sum_{x \in T} d_{G-S}(x)-f(T) \geq \max _{U \subseteq S, H \subseteq E(G-U),|U|=n^{\prime},|H|=m}\left\{g(U)-e_{H}(S, T)+\sum_{x \in T} d_{H}(x)\right\},
$$

for any two non-disjoint subsets $T, S \subseteq V(G)$ with $|S| \geq n^{\prime}$.

By setting $n^{\prime}=0$ in Lemma 4 , the sufficient and necessary condition for a graph to be all fractional $(g, f, m)$-deleted can be immediately derived.

Lemma 5 Assume $m$ is a nonnegative integer. Set $g, f: V(G) \rightarrow \mathbb{Z}^{+}$as integer-valued functions having $g(x) \leq f(x)$ for every $x \in V(G)$, and $H$ as a subgraph of $G$ contains $m$ edges. Then $G$ is all fractional $(g, f, m)$-deleted iff

$$
g(S)+\sum_{x \in T} d_{G-S}(x)-f(T) \geq \sum_{x \in T} d_{H}(x)-e_{H}(S, T),
$$

for any non-disjoint subsets $T$ and $S$ of $V(G)$. 
Using the same approach, the concepts of all fractional ID- $(g, f, m)$-deleted graph (or, all fractional independent-set-deletable $(g, f, m)$-deleted graph), all fractional ID- $(g, f)$ factor-critical graph can be defined as well. By setting $f(x)=b$ and $g(x)=a$, these concepts reduce to all fractional ID- $(a, b, m)$-deleted graph and all fractional ID- $[a, b]$-factor-critical graph, respectively.

It's easy to see that the necessary and sufficient conditions of the fractional critical deleted graphs and all fractional critical deleted graphs share a lot of similarities in their expressions, so it has inspired us to check it the proofs Theorems 1-3 above can also be adapted to obtain the corresponding result in all fractional setting. In fact, such conclusions can be gained easily.

Theorem 4 Assume $G$ is a graph, and $b, a, n^{\prime}, m$ are nonnegative integers. Set $g, f$ as integer-valued functions on $V(G)$ such that $1 \leq a \leq g(x) \leq f(x) \leq b$ for every vertex $x$ in G. Then $G$ is all fractional $\left(g, f, n^{\prime}, m\right)$-critical deleted if $\kappa(G) \geq \max \left\{\frac{(b+1)^{2}}{2}+\frac{2 m}{a}+\right.$ $\left.n^{\prime}, \frac{(b+1)^{2} \alpha(G)+4\left(a n^{\prime}+2 m\right)}{4 a}\right\}$.

Theorem 5 Assume $G$ is a graph, and $b, a, m$ are nonnegative integers. Set $g, f$ as two integer-valued functions on $V(G)$ such that $1 \leq a \leq g(x) \leq f(x) \leq b$ for every vertex $x$ in $G$. Then $G$ is all fractional $I D-(g, f, m)$-deleted if

$$
\alpha(G) \leq \frac{4(a(\delta(G)+1-b)-2 m)}{4 a+(1+b)^{2}} .
$$

Theorem 6 Assume $G=(X, Y ; E(G))$ is a bipartite graph, $b$, a are two positive integers with $1 \leq a \leq b$, and functions $g, f$ are integer-valued on $V(G)$ such that $a \leq g(x) \leq f(x) \leq b$ for each vertex $x$ in $G$. Let $n=|X|=|Y|>\frac{(b+a-2)(a+2+b)}{4 a}, f(X)=f(Y)$ and $g(X)=g(Y)$. Then $G$ admits an all fractional $(g, f)$-factor if $I_{B}(G)>\frac{n-1}{2 \sqrt{a n+1}-b-a}$.

We discover that in the proofs of theorems, the first step is achieved in the following way:

$$
\begin{aligned}
a|S| & +\sum_{x \in T} d_{G-S}(x)-b|T|-a n^{\prime}-\left(\sum_{x \in T} d_{H}(x)-e_{H}(S, T)\right) \\
& \leq\left(|S|-n^{\prime}\right) a-\left(\sum_{x \in T} d_{H}(x)+\sum_{x \in T}\left(d_{G-S}(x)-b\right)-e_{H}(S, T)\right) \\
& \leq g(S-U)+\sum_{x \in T}\left(d_{G-S}(x)-f(x)\right)-\left(\sum_{x \in T} d_{H}(x)-e_{H}(S, T)\right) \leq-1 .
\end{aligned}
$$

Therefore, the remaining steps of the proofs are the similar to those in Sect. 2 (set $\Delta=0$ ) except that $b n^{\prime}$ is replaced by $a n^{\prime}$ for the critical part. This is why the above three theorems can be obtained directly.

\section{Conclusions and future works}

In this paper, we emphasized the relationship between graph parameters and the existence of a fractional $(g, f)$-factor in different settings. Sufficient conditions were given 
in terms of connectivity, independence number, and isolated toughness. Moreover, the sharpness of the first two conclusions was illustrated by constructing special graph classes.

Although there have been several recent advances related to the existence of fractional factor in various settings of this problem, the study of generalization properties of factional factors has been largely limited to special settings. The bipartite graph setting of the fractional factor problem is perhaps the most important one, and, in fact, only one theoretical result has been derived for it. We believe this subject could be deeply studied in the future, and the following open problem is raised here.

Problem 1 What is the necessary and sufficient condition for fractional deleted graph and fractional critical graph in the bipartite graph setting? Can isolated toughness condition in Theorem 3 extend to a graph to be fractional $\left(g, f, n^{\prime}\right)$-critical or to be fractional $(g, f, m)$ deleted?

Note that the parameter $\Delta$, to a certain extent, represents the difference between the two functions $g$ and $f$. When it comes to the fractional factor applications in computer networks, $\Delta$ expresses the width of the packet size that the network site can bear. Obviously, this is a very important parameter which deserves further study. In the fractional $(g, f)$-factor setting, we see that in the first step of proofs, we always use the inequality $(a+\Delta)|S|+d_{G-S}(T)-|T|(b-\Delta) \leq f(S)-g(T)+d_{G-S}(T)$, and in this way $\Delta$ can be considered in the final obtained conditions. However, in the all fractional $(g, f)$-factor setting, in view of Lemma 4, this inequality becomes $a|S|+d_{G-S}(T)-b|T| \leq f(S)-g(T)+d_{G-S}(T)$. It seems that parameter $\Delta$ can't participate in the derivation process, and this is the reason why in Sect. 4 we need to skip $\Delta$ in the discussion. Intuition tells us that if we use an appropriate trick, $\Delta$ would participate in the calculation, and this is what we need to consider in the future.

Problem 2 How to make parameter $\Delta$ work in the all fractional $(g, f)$-factor setting?

Funding

This work has been partially supported by Ministerio de Ciencia, Innovación y Universidades from Spanish Government (No. PGC2018-097198-B-I00) and Fundación Séneca de la Región de Murcia grant number 19219/PI/14, and National Science Foundation of China grant number 11761083.

Availability of data and materials

The authors declare that this paper has been developed without any data.

Competing interests

We declare that none of the authors have any competing interests in the manuscript.

Authors' contributions

The authors have worked equally when writing this paper. All authors read and approved the final manuscript.

Author details

'Departamento de Matemática Aplicada y Estadística, Hospital de Marina, Universidad Politécnica de Cartagena, Cartagena, Spain. ${ }^{2}$ School of Information Science and Technology, Yunnan Normal University, Kunming, China.

\section{Publisher's Note}

Springer Nature remains neutral with regard to jurisdictional claims in published maps and institutional affiliations. 


\section{References}

1. Bian, Q.J., Zhou, S.Z.: Independence number, connectivity and fractional $(g, f)$-factors in graphs. Filomat 29(4), 757-761 (2015)

2. Bloem-Reddy, B., Orbanz, F.: Random-walk models of network formation and sequential Monte Carlo methods for graphs. J. R. Stat. Soc. B 80(5), 871-898 (2018)

3. Bondy, J.A., Murty, U.S.R.: Graph Theory. Springer, Berlin (2008)

4. Bukhari, J., Park, J.H., Yoon, W.: Providing multicast services over SDN-evolved LTE network: architecture, procedures and performance analysis. Comput. Commun. 127, 131-145 (2018)

5. Folkman, J., Fulkerson, D.R.: Flows in infinite graphs. J. Comb. Theory 8, 30-44 (1970)

6. Gao, W.: Some results on fractional deleted graphs. Doctoral dissertation, Soochow University (2012)

7. Gao, W., Dimitrov, D., Abdo, H.: Tight independent set neighborhood union condition for fractional critical deleted graphs and ID deleted graphs. Discrete Contin. Dyn. Syst., Ser. S 12, 711-721 (2019)

8. Gao, W., Guirao, J.L.G., Abdel-Aty, M., Xi, W.F.: An independent set degree condition for fractional critical deleted graphs. Discrete Contin. Dyn. Syst., Ser. S 12, 877-886 (2019)

9. Gao, W., Guirao, J.L.G., Chen, Y.J.: A toughness condition for fractional $(k, m)$-deleted graphs revisited. Acta Math. Appl. Sin. Engl. Ser. (in press)

10. Gao, W., Guirao, J.L.G., Wu, H.L.: Two tight independent set conditions for fractional $(g, f, m)$-deleted graphs systems. Qual. Theory Dyn. Syst. 17(1), 231-243 (2018)

11. Gao, W., Liang, L., Chen, Y.H.: An isolated toughness condition for graphs to be fractional ( $k, m)$-deleted graphs. Util. Math. 105, 303-316 (2017)

12. Gao, W., Liang, L., Xu, T.W., Zhou, J.X.: Degree conditions for fractional $\left(g, f, n^{\prime}, m\right)$-critical deleted graphs and fractional ID- $(g, f, m)$-deleted graphs. Bull. Malays. Math. Sci. Soc. 39, 315-330 (2016)

13. Gao, W., Wang, W.F.: New isolated toughness condition for fractional $(g, f, n)$-critical graphs. Colloq. Math. 147, 55-66 (2017)

14. Gao, W., Zhang, Y.Q., Chen, Y.J.: Neighborhood condition for all fractional $\left(g, f, n^{\prime}, m\right)$-critical deleted graphs. Open Phys. 16, 544-553 (2018)

15. Ikarashi, H., Jin, Y., Yamai, N., Kitagawa, N., Okayama, K.: Design and implementation of SDN-based proactive firewall system in collaboration with domain name resolution. IEICE Trans. Inf. Syst. E101.D, 2633-2643 (2018)

16. Kaur, D., Aujla, G.S., Kumar, N., Zomaya, A.Y., Perera, C., Ranjan, R.: Tensor-based big data management scheme for dimensionality reduction problem in smart grid systems: SDN perspective. IEEE Trans. Knowl. Data Eng. 30(10), 1985-1998 (2018)

17. Li, Z.P., Zhang, X.S., Yan, G.Y.: Isolated toughness and fractional k-deleted graphs. OR Trans. 7(4), $79-85$ (2003)

18. Liu, D.L., Wang, C.X., Wang, S.H.: Hamilton-connectivity of interconnection networks modeled by a product of graphs. Appl. Math. Nonlinear Sci. 3, 419-426 (2018)

19. Maity, I., Mondal, A., Misra, S., Mandal, C.: CURE: consistent update with redundancy reduction in SDN. IEEE Trans. Commun. 66(9), 3974-3981 (2018)

20. Nghe, P., Mulder, B.M., Tans, S.J.: A graph-based algorithm for the multi-objective optimization of gene regulatory networks. Eur. J. Oper. Res. 270(2), 784-793 (2018)

21. Nielsen, J.B., Pedersen, E.: On the modelling of heat exchangers and heat exchanger network dynamics using bond graphs. Math. Comput. Model. Dyn. Syst. 24(6), 626-642 (2018)

22. Ore, O.: Studies on directed graphs, I. Ann. Math. 63(3), 383-406 (1956)

23. Ore, O.: Studies on directed graphs II. Ann. Math. 64(1), 142-153 (1956)

24. Ore, O.: Studies on directed graphs, III. Ann. Math. 68(3), 526-549 (1958)

25. Sadik, M., Akkari, N., Aldabbagh, G.: SDN-based handover scheme for multi-tier LTE/Femto and D2D networks. Comput. Netw. 142, 142-153 (2018)

26. Sahoo, K.S., Puthal, D., Tiwary, M., Rodrigues, J.J.P.C., Sahoo, B., Dash, R.: An early detection of low rate DDoS attack to SDN based data center networks using information distance metrics. Future Gener. Comput. Syst. 89, 685-697 (2018)

27. Salman, O., Elhajj, J., Chehab, A., Kayssi, A.: IoT survey: an SDN and fog computing perspective. Comput. Netw. 143 221-246 (2018)

28. Satchou, G.A.K., Anoh, N.G., N'Takpe, T., Oumtanaga, S.: Optimization of the latency in networks SDN. Int. J. Comput. Commun. Control 13(5), 824-836 (2018)

29. Shah, S.A.R., Wu, W.J., Lu, Q., Zhang, L., Sasidharan, S., DeMar, P., Guok, C., Macauley, J., Pouyoul, E., Kim, J., Noh, S.Y.: AmoebaNet: an SDN-enabled network service for big data science. J. Netw. Comput. Appl. 119, 70-82 (2018)

30. Trentin, E., Di lorio, E.: Nonparametric small random networks for graph-structured pattern recognition. Neurocomputing 313, 14-24 (2018)

31. Vizarreta, P., Trivedi, K., Helvik, B., Heegaard, P., Blenk, A., Kellerer, W., Machuca, C.M.: IEEE Trans. Netw. Serv. Manag. 15(3), 1090-1104 (2018)

32. Yang, J.B., Ma, Y.H., Liu, G.Z.: Fractional (g, f)-factors in graphs. Appl. Math. J. Chin. Univ. Ser. A 16, $385-390$ (2001)

33. Yuan, Y., Hao, R.X.: Independence number, connectivity and all fractional $(a, b, k)$-critical graphs. Discuss. Math., Graph Theory 39, 183-190 (2019)

34. Zhou, S.Z.: Remarks on orthogonal factorizations of digraphs. Int. J. Comput. Math. 91(10), 2109-2117 (2014)

35. Zhou, S.Z: Some results about component factors in graphs. RAIRO Oper. Res. (in press). https://doi.org/10.1051/ro/2017045

36. Zhou, S.Z., Duan, Z.M., Pu, B.Y.: Isolated toughness and fractional ( $g, f)$-factors of graphs. Ars Comb. 110, 239-247 (2013)

37. Zhou, S.Z., Liu, H.X., Zhang, T.: Randomly orthogonal factorizations with constraints in bipartite networks. Chaos Solitons Fractals 112, 31-35 (2018)

38. Zhou, S.Z., Pan, Q.R.: An isolated toughness condition for graphs to be fractional $(a, b, k)$-critical graphs. Util. Math. 92 , 251-260 (2013)

39. Zhou, S.Z., Sun, Z.R.: Neighborhood conditions for fractional ID-k-factor-critical graphs. Acta Math. Appl. Sin. Engl. Ser. 34(3), 636-644 (2018)

40. Zhou, S.Z., Sun, Z.R., Xu, Z.R.: A result on r-orthogonal factorizations in digraphs. Eur. J. Comb. 65, 15-23 (2017) 
41. Zhou, S.Z., Xu, L., Xu, Y.: A sufficient condition for the existence of a $k$-factor excluding a given $r$-factor. Appl. Math. Nonlinear Sci. 2, 13-20 (2017)

42. Zhou, S.Z., Xu, L., Xu, Z.R.: Remarks on fractional ID-k-factor-critical graphs. Acta Math. Appl. Sin. Engl. Ser. 35(2), 458-464 (2019)

43. Zhou, S.Z., Yang, F., Xu, L.: Two sufficient conditions for the existence of path factors in graphs. Sci. Iran. (2018). https://doi.org/10.24200/SCI.2018.5151.1122

Submit your manuscript to a SpringerOpen ${ }^{\circ}$ journal and benefit from:

- Convenient online submission

$\checkmark$ Rigorous peer review

- Open access: articles freely available online

- High visibility within the field

- Retaining the copyright to your article

Submit your next manuscript at $\boldsymbol{~ s p r i n g e r o p e n . c o m ~}$ 